\title{
CrystEngComm
}

Check for updates

Cite this: CrystEngComm, 2017, 19, 6443

Received 27th August 2017,

Accepted 9th October 2017

DOI: $10.1039 / c 7 c e 01558 e$

rsc.li/crystengcomm

\section{Type I vs. quasi-type II modulation in CdSeaCdS tetrapods: ramifications for noble metal tipping $\dagger$}

\author{
Nicholas G. Pavlopoulos, ${ }^{a}$ Jeffrey T. Dubose, ${ }^{a}$ Yawei Liu, ${ }^{b}$ Xing Huang, (D) ${ }^{c}$ \\ Nicola Pinna, iD d Marc-Georg Willinger, ${ }^{c}$ Tianquan Lian, ${ }^{b}$ \\ Kookheon Char ${ }^{\mathrm{e}}$ and Jeffrey Pyun iD *ae
}

\begin{abstract}
We report on noble metal tipping of heterostructured nanocrystals (NCs) of CdSe@CdS tetrapods (TPs) as a chemical reaction to manifest energetic differences between type I and quasi-type II heterojunctions. The energetics between zincblende (ZB) CdSe seed and wurtzite CdS TP arms has previously been probed primarily using ultrafast spectroscopic methods. However, to interrogate the energetics of CdSe@CdS TPS from both larger and very small ZB CdSe seeds (i.e., effective diameters $\left(D_{\text {eff }}\right) 6.2$ to $1.8 \mathrm{~nm}$ ) we utilize the photodeposition of gold nanoparticles as a facile and selective chemical reaction to correlate reaction rates with tetrapod energetics. We observe a 20 -fold enhancement in the rate of selective photodeposition of a single AuNP tip onto this series of TPs, which were attributed to straddling of type I vs. quasi-type II energetics as a direct consequence of varying ZB CdSe seed size. Synthetic access to type I and quasi-type II TPs enabled the synthesis of singly tipped AuNP-CdSeaCdS TPs in gram quantities, which is the largest scale synthesis of metal-semiconductor Janus nanoparticles to date.
\end{abstract}

\section{Introduction}

II-VI semiconductor nanocrystals are promising nanomaterials for optoelectronics, mechanical sensing, self-assembly, and photocatalysis. ${ }^{1-17}$ Of these, colloidal CdSe@CdS core@shell materials have been studied for their unique optical and electronic properties. Consisting of a small CdSe quantum dot (QD) embedded within a CdS shell of tunable dimensions, exquisite levels of control over nanocrystal energetics (type I vs. quasi-type II) and shape (sphere, nanorod, tetrapod, octapod) have been demonstrated to date. ${ }^{18-22}$ Whereas energetics can principally be tuned by individual component dimensions, particle shape has been found to be primarily dependent on the CdSe-core crystal structure, with the more stable wurtzite (W) structure resulting in nanorods (NR), and the less stable zinc blende (ZB) structure affording

\footnotetext{
${ }^{a}$ Department of Chemistry and Biochemistry, University of Arizona, 1306 East University Boulevard, Tucson, Arizona 85721, USA. E-mail: jpyun@email.arizona.edu ${ }^{b}$ Department of Chemistry, Emory University, 1515 Dickey Drive, NE, Atlanta, 30322 Georgia, USA

${ }^{c}$ Institut fur Chemie, Humboldt-Universitat zu Berlin, Brook-Taylor-Staße 2, 12489 Berlin, Germany

${ }^{d}$ Department of Inorganic Chemistry, Fritz Haber Institute of the Max Planck Society, Berlin, Germany

${ }^{e}$ World Class University Program for Chemical Convergence for Energy and Environment, School of Chemical and Biological Engineering, Seoul National University, Seoul 151-744, Korea

$\dagger$ Electronic supplementary information (ESI) available. See DOI: 10.1039/ c7ce01558e
}

access to tetrapods (TPs). Among these II-VI semiconductor heterostructures, nanorod systems have been studied extensively for directed charge transfer, for which solution deposited metallic nanoparticle "tips" can be energetically aligned to promote electron transfer from photogenerated excitons in the semiconductor phase. ${ }^{23-35}$ Such asymmetrically metaltipped semiconductor nanocrystals (NCs) were pioneered by Banin et al., where the seminal NR systems were terminally tipped with either $\mathrm{Au}$ or Pt nanoparticles. ${ }^{36-39}$ More recently, CdSe seeded CdS (CdSe@CdS) TPs with noble metal NP tips have been developed, in particular with high selectivity to enable photodeposition of a single AuNP tip on the end of one arm of each tetrapod. ${ }^{40,41}$

An attractive feature that has been exploited for CdSe@CdS NRs was the ability to modulate the energetics of the heterostructure junctions from type I (with lowest energy holes and electrons localized in the CdSe core) to quasi-type II (with lowest energy holes localized in the CdSe core and lowest energy electrons delocalized across the CdSe/CdS components) by variation of the $\mathrm{W}$ CdSe NC seed size. ${ }^{42-50}$ This phenomenon has been extensively investigated and revealed that control of the CdSe NC dimensions near the $2.8 \mathrm{~nm}$ size regime (effective diameter) was the critical size range to access the transition from type I to quasi-type II energetics: below $2.8 \mathrm{~nm}$ core sizes, quasi-type II behavior dominates, whereas sizes above $2.8 \mathrm{~nm}$ result in a type I band alignment. ${ }^{49,51}$ While more challenging to prepare than nanorods systems, CdSe@CdS tetrapods have been studied for stress- 
sensing, photovoltaic, and LED applications in recent years. $^{1,3,12,16,52}$ Particularly, the seminal work of Alivisatos and Chan have reported on the synthesis and spectroscopic characterization of CdSe@CdS TPs with $4.0 \mathrm{~nm}$ and $3.0 \mathrm{~nm}$ zincblende CdSe NC seeds, respectively, leading to applications in mechanical stress sensing and dual emission lightemitting diodes. ${ }^{1,3,12,52}$

These studies pointed to the possibility of modulating energetics of CdSe@CdS TPs by variation of ZB CdSe NC size from 2-6 $\mathrm{nm}$. However, synthetic accessibility to both welldefined ZB CdSe NC sizes and subsequent CdSe@CdS TPs have limited further studies to enable correlation of type I vs. quasi-type II energetics with ZB CdSe seed size. Specifically, while CdSe@CdS nanorod systems demonstrate enhanced charge separation (and increased catalytic performance) with core sizes below $2.8 \mathrm{~nm},{ }^{23,48,49,51}$ the effect of ZB CdSe core size on overall tetrapod energetics (type I vs. quasi-type II) has yet to be probed below the $3.5 \mathrm{~nm}$ core-size regime spectroscopically or chemically. Additionally, synthetic access to CdSe@CdS TPs in this size regime would be desirable as these have not been utilized for metal tipping processes and would be expected to be more reactive due to enhanced charge dissociation, affording more electrons for noble metal reduction. In our earlier example for selective photodeposition of a single AuNP onto CdSe@CdS TPs, larger 6 nm ZB CdSe seeds were used, which presumably possessed type I band edge offsets, requiring long reaction times $(\sim 1 \mathrm{~h})$ and being limited to small synthetic scale $(8 \mathrm{mg} \mathrm{Au}-$ CdSe@CdS TP). ${ }^{40,41}$

Herein, we report on the use of noble metal photodeposition onto CdSe@CdS TPs as a chemical probe to map the intrinsic reactivity differences that arise from type I vs. quasi-type II energetics in these heterostructured materials. While the preparation of metal-semiconductor nanorod and tetrapod materials has been extensively reported, the exploitation of differences in metal tipping reaction kinetics to manifest chemical reactivity enhancements as a consequence of TP energetics has not been exploited. Furthermore, to enable these studies, a colloidal total synthesis was devised using a new combination of related synthetic methods to prepare a series of CdSe@CdS TPs with varying ZB CdSe seed sizes to enable structure-activity correlations arising from type I vs. quasi-type II energetics. The key synthetic steps that required optimization in this total synthesis were the preparation of ZB CdSe NCs over a wide range of sizes (particularly for very small NC diameters, $<3 \mathrm{~nm}$ ) and the development of incremental addition reactions to use these ZB CdSe seeds for the preparation of well-defined CdSe@CdS TPs. Profoundly enhanced rates of AuNP photodeposition onto CdSe@CdS TP as a function of using small CdSe NC seeds $\left(D_{\text {eff }}<2.8 \mathrm{~nm}\right)$ were observed, which was attributed to the onset of quasi-type II energetics in CdSe@CdS TPs. By using these more reactive TPs, the synthesis of Janus nanoparticles composed of AuNP-tipped CdSe@CdS TPs was conducted on very large scale for these types of materials $(2.5 \mathrm{~g})$, using only ambient solar irradiation for rapid and selective AuNP photo- deposition, which represents the largest scale synthesis of metal-semiconductor Janus nanoparticles to date.

\section{Experimental}

\section{Chemicals}

All chemicals were commercially available and used as received, unless otherwise noted. 1-Octadecene (ODE, 90\%) was purchased from Acros. Gold(III) chloride $\left(\mathrm{AuCl}_{3}, 99.99 \%\right)$, cadmium oxide (CdO, 99.998\%), hexadecylamine (HDA, 98\%), myristic acid (99.99\%), and elemental sulfur (99.5\%) were purchased from Alfa Aesar. Cadmium(II) acetylacetonate $\left(\mathrm{Cd}(\mathrm{acac})_{2}\right) \quad(99.9 \%)$, didodecyldimethylammonium bromide (DDAB, 98\%), oleic acid (OLAC, 90\%), and oleylamine (OLAM, 70\%) were purchased from Sigma Aldrich. Octadecylphosphonic acid (ODPA) (97\%), elemental selenium (99.99\%), trioctylphosphine (TOP) (97\%), and trioctylphosphine oxide (TOPO) (90\%) were purchased from Strem. absolute ethanol was commercially available from Decon Labs. Acetone, methanol, isopropanol, and toluene were commercially available from Fisher Scientific.

\section{Representative synthesis of zincblende CdSe NCs (1.8-8.0 nm)}

Inspired by the methods of Hens, a hot-injection approach was utilized to prepare small ZB CdSe nanocrystals. In a 250 $\mathrm{mL}$ three neck round bottom flask, Cd(acac) ${ }_{2}$ (248.5 mg; $0.800 \mathrm{mmol})$ and OLAC (1.02 g; $1.13 \mathrm{~mL} ; 3.60 \mathrm{mmol})$ were degassed at $50{ }^{\circ} \mathrm{C}$ for 45 minutes followed by heating to 150 ${ }^{\circ} \mathrm{C}$ under Ar. This stock was then cooled to room temperature by injection of 1-ODE (30 mL), followed by addition of HDA $(1.12 \mathrm{~g}, 4.80 \mathrm{mmol})$ and ODA $(0.040 \mathrm{~g}, 0.15 \mathrm{mmol})$, and degassing of the flask. Subsequently, the mixture was heated under Ar to $170{ }^{\circ} \mathrm{C}$ at which point a $1.0 \mathrm{M}$ TOP-selenide stock solution $(8.0 \mathrm{~mL})$ was injected. The reaction was quenched after 10 seconds by hexanes injection, resulting in $2.0 \mathrm{~nm} \mathrm{ZB}$ CdSe NCs (see Fig. $\mathrm{S} 1 \uparrow$ for detailed quench time/particle size correlations). Detailed synthetic methods for the preparation of larger ZB CdSe NCs $(2.8-8.0 \mathrm{~nm})$ are included in the ESI. $\dagger$

\section{Synthesis of CdSe@CdS tetrapods by incremental injection}

The preparation of CdSe@CdS tetrapods of tunable band alignment was achieved with an incremental hot-injection approach. In a $100 \mathrm{~mL}$ three neck round bottom flask, TOPO (5.40 g; $140 \mathrm{mmol})$, ODPA (285 mg; $0.852 \mathrm{mmol})$, CdO (110 $\mathrm{mg} ; 0.857 \mathrm{mmol}$ ), and OLAC (912 $\mathrm{mg} ; 1.02 \mathrm{~mL} ; 3.63 \mathrm{mmol}$ ) were degassed at $150{ }^{\circ} \mathrm{C}$ for 30 minutes followed by heating to $300{ }^{\circ} \mathrm{C}$. At this point, TOP $(1.01 \mathrm{~g}, 1.22 \mathrm{~mL}, 3.73 \mathrm{mmol})$ was injected to the flask, and the reaction mixture was wrapped with cotton and stabilized at $350{ }^{\circ} \mathrm{C}$ for 30 minutes. Next, a $1.7 \mathrm{mmol}$ stock of ZB CdSe QDs of the desired size in TOP was swiftly injected to the flask, followed immediately by a $0.55 \mathrm{M}$ TOP-sulfide stock solution $(0.20 \mathrm{~mL})$. The reaction was allowed to proceed for 3 minutes at $350{ }^{\circ} \mathrm{C}$ to facilitate growth of the tetrapod shell. This process was repeated three additional times $(0.80 \mathrm{~mL}$ TOP $=\mathrm{S}$ injected, 12 minutes 
total reaction time), after which the reaction was quenched by removal of the heating mantle.

\section{Synthesis of Au-CdSe@CdS tetrapods}

To an evacuated and backfilled 1-dram vial was added a 0.325 $\mathrm{mM}$ stock of synthesized tetrapods in toluene $(0.68 \mathrm{~mL})$, followed by a solution of $8.80 \mathrm{mM} \mathrm{AuCl}_{3}$ and $9.01 \mathrm{mM} \mathrm{DDAB}$ in toluene $(0.25 \mathrm{~mL})$. The resulting solution was irradiated at $365 \mathrm{~nm}$ in a custom-built photochemical reactor between 2.5 minutes to 1 hour, depending on the CdSe core size (see Fig. 5), resulting in singly-tipped Au-TP Janus nanoparticles. Detailed synthetic methods for the synthesis of Au-TP Janus nanoparticles on $2.5 \mathrm{~g}$ scale are included in the ESI.†

\section{Characterization}

UV-vis measurements were obtained using an Agilent 8453 spectrophotometer. Fluorescence measurements were obtained using a FL3-11 Fluorolog-3 Spectrofluorometer (Jobin Yvon-Spex Instruments SA). Low resolution bright field TEM images (all not specifically labeled "high resolution") were obtained using a Technai Spirit transmission electron microscope (FEI) at $120 \mathrm{kV}$ or a CM200 $\mathrm{LaB}_{6}$ (Philips) at $200 \mathrm{kV}$ using in-house prepared carbon coated copper grids $(\mathrm{Cu}$, hexagon, $300 \mathrm{mesh}$ ). Image analysis was performed using ImageJ software (Rasband, W.S., National Institutes of Health, http:// rsb.info.nih.gov/ij/) from bright field TEM images at a minimum of $110000 \times$ magnification by sizing a minimum of 100 nanoparticles. High-resolution TEM (HRTEM), bright field scanning TEM (STEM), high angle annular dark field STEM (HAADF-STEM) images and EDX maps were obtained using a JEM-ARM200F (Jeol) double-corrected Cold Field Emission TEM operated at $200 \mathrm{kV}$. Thermogravimetric analysis was carried out under nitrogen atmosphere using a TGA Q50 instrument and software from TA Instruments. SEM images were taken on a Hitachi 4800 FE-SEM (30 kV accelerating voltage) on the as-prepared samples (no metallic overcoating).

\section{Results and discussion}

The first step in this broader study was the development of a colloidal total synthesis ${ }^{47,53,54}$ for the various materials prepared in this work, which consisted of the following: (1) development of modified synthetic methods to prepare welldefined ZB CdSe seed nanocrystals in the size range from 2$8 \mathrm{~nm}$, (2) a comprehensive synthetic approach to utilize these ZB CdSe NCs (particularly of small sizes below $3 \mathrm{~nm}$ ) to prepare well-defined CdSe@CdS tetrapods with tunable energetics between type I vs. quasi-type II junctions, and (3) the design and use of quasi-type II CdSe@CdS TPs synthesized from ultrasmall $(2 \mathrm{~nm})$ seeds to accelerate the kinetics of the selective photodeposition of a single AuNP tip $(D \sim 7 \mathrm{~nm})$ per tetrapod. Insights from these new advances enabled the multi-gram synthesis of singly tipped Au-CdSe@CdS TPs using sunlight as the only irradiation source for photodeposition (which occurred in under $90 \mathrm{~s}$ ).
Preparation and characterization of well defined zincblende CdSe nanocrystals of tunable size (1.8-8.0 nm)

While the preparation of well-defined $\mathrm{W}$ CdSe nanocrystals have been successful over a wide range of particle sizes $(\sim 2-6$ $\mathrm{nm}$ ) by variation of reaction time for standard hot injection approaches, ${ }^{28,47}$ similar approaches cannot be readily applied to prepare $\mathrm{ZB}$ CdSe NCs due to the reduced stability of the zincblende phase, particularly for smaller NCs $(D<3.0 \mathrm{~nm})$. The methods of Hens provided access to ZB CdSe NCs of varying seed sizes $(2.2-4.9 \mathrm{~nm})$ in yields suitable for optical studies, ${ }^{55}$ but while variables affecting size were identified (reaction time, temperature, precursor reactivity), detailed methods for tuning reaction scale were not extensively discussed. In a global sense, the synthesis of ZB CdSe NCs as precursors specifically for use in the synthesis of tetrapod heterostructures has been limited to certain sizes $(D \sim 3.5-6.0 \mathrm{~nm}) .{ }^{14,56}$

Consequently, as the first step of the tetrapod synthesis, ZB CdSe seed syntheses were employed using methods inspired by the work of Alivisatos, Cao, Hens, and our previous studies to allow for the preparation of well-defined NCs ranging in effective diameters from 2-8 $\mathrm{nm}$. Synthetic methods were investigated to afford materials on much larger scales ( 200-500 $\mathrm{mg}$ ) than previously demonstrated for ZB CdSe NCs. Due to the reduced phase stability of ZB CdSe at smaller particle sizes, the use of four different procedures for preparing ZB CdSe NCs of varying sizes were required, each using different formulations of cadmium and selenium precursors, along with ligand mixtures.

For large particle sizes (5.5-8.0 nm), methods based off of our previously published work for $6 \mathrm{~nm}$ ZB CdSe NCs were utilized where near stoichiometric amounts of unpassivated selenium (selenium-octadecene suspension) and cadmium acetylacetonate precursor were reacted through hot injection methods in the presence of stabilizing ligands to generate $\mathrm{ZB}$ CdSe NCs (Scheme 1d). ${ }^{40,41}$ In this synthetic approach, oleic acid was utilized as the stabilizing ligand, and annealing

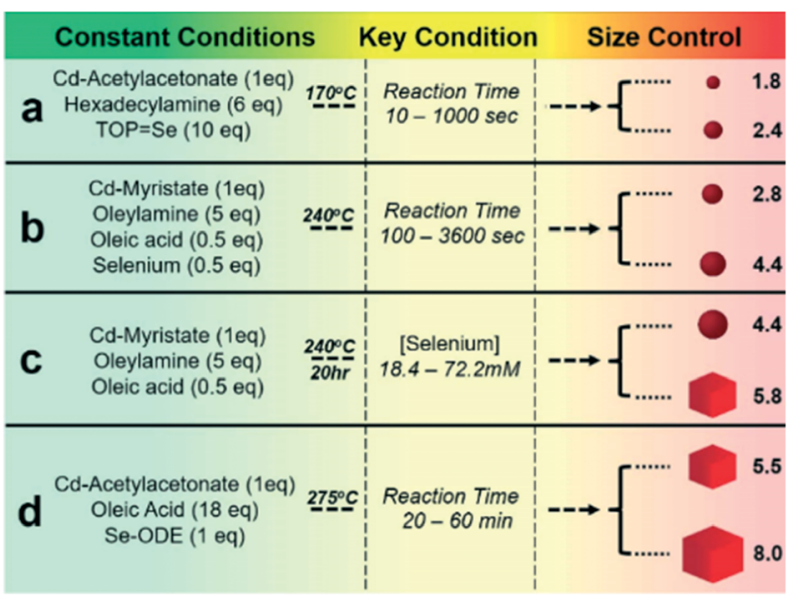

Scheme 1 Schematic showing the constant and key tunable parameters utilized (a) to access 1.8-2.4 nm zincblende CdSe NCs, (b) to access 2.8$4.4 \mathrm{~nm}$ zincblende CdSe NCs, (c) to access 4.4-5.8 nm zincblende CdSe $\mathrm{NCs}$, and (d) to access 5.5-8.0 nm zincblende CdSe NCs. 
at $275{ }^{\circ} \mathrm{C}$ for $20-60$ minutes resulted in $\mathrm{ZB}$ CdSe nanocubes of 5.5-8.0 nm size. For ZB CdSe NCs with diameters between 2.8-5.8 $\mathrm{nm}$, injection-free methods were utilized, wherein a selenium-octadecene suspension was reacted with excess cadmium myristate synthesized in situ at $240{ }^{\circ} \mathrm{C}$ (Scheme 1b and c). ${ }^{14,56}$ From this method, to access ZB CdSe NCs with particle sizes of 4.4-5.8 nm, we employed a 20 hour annealing step at $240{ }^{\circ} \mathrm{C}$ to prepare uniform NCs of predominantly cubic crystal habit, with particle size control being afforded through variation of selenium concentration (18.4$72.2 \mathrm{mM}$ ) (Scheme 1c). Alternatively, it was found that smaller ZB CdSe sizes between $2.8-4.4 \mathrm{~nm}$ could be obtained under conditions of constant selenium concentration (72.4 $\mathrm{mM}$ ) and variable reaction time (100-3600 seconds) (Scheme 1b). Access to ZB CdSe NCs in the ultrasmall size regime (1.8-2.4 $\mathrm{nm}$ ) was achieved through modifications to the approaches of Hens (Scheme 1a) ${ }^{55}$ For the synthesis of tetrapod nanocrystals, key modifications were made to the published procedures to allow for reproducible and scalable access to ZB CdSe NCs below $2.5 \mathrm{~nm}$ in effective diameter. By maintaining a constant reaction temperature $\left(170{ }^{\circ} \mathrm{C}\right)$, and using reaction time as the sole handle for particle size (10-600 seconds), we found that the yield was significantly improved (up to $275 \mathrm{mg}$ isolated yield) while allowing for highly reproducible size control in the regime of $1.8-2.4 \mathrm{~nm}$. It should be noted that larger ZB CdSe NCs $(D \sim 3-8 \mathrm{~nm})$ maintained excellent colloidal stability during preparation and isolation steps. Conversely, very small CdSe NCs $(D<3$ $\mathrm{nm}$ ) showed significant shifts/broadening of the low energy absorption transitions in solution after subjection to our isolation techniques, presumably due to partial corrosion induced from excess ligands and perturbations incurred from centrifugation (irreversible aggregation). To circumvent this, modified conditions were designed to remove excess $\mathrm{Cd} / \mathrm{Se}$ species while maintaining dispersion of ultrasmall ZB CdSe NCs. In this case, a high boiling point non-coordinating solvent containing a small amount of carboxylic acid ligand (ODE/OLAC) was added to the nanocrystal pellet after each purification step (see ESI $\dagger$ ). Upon storage in an argon-filled glove box, particles purified via these methods exhibited no absorption broadening or variations in reactivity over the course of multiple months.

Transmission electron microscopy (TEM) studies of the ZB CdSe seeds were conducted to confirm uniformity of particle size, morphology, and crystallinity (Fig. 1). Bright field TEM confirmed the ability to prepare well-defined nanocrystals ranging from 2-8 $\mathrm{nm}$ (with size deviations below 10\%), where larger 5.5-8.0 $\mathrm{nm}$ nanocrystals were observed to adopt more cubic morphologies, while smaller particles exhibited more spherical morphology. Electron diffraction patterns of CdSe NCs were observed to exhibit prominent (111), (220), and (311) reflections characteristic of ZB CdSe (Fig. S8 $\dagger$ ). ${ }^{57}$ It should be noted that while electron diffraction mapping was performed for CdSe NPs below $2.5 \mathrm{~nm}$ in size, broad reflections owing to the small size of the nanocrystals were observed. Nonetheless, the complete absence of WZ CdSe (002),

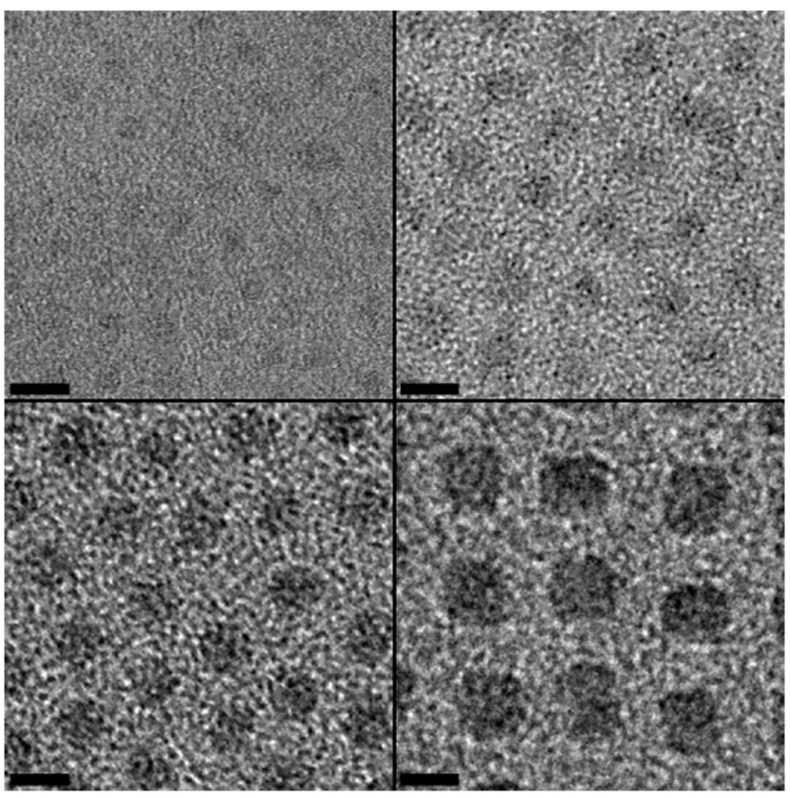

Fig. 1 High resolution TEM images of zincblende CdSe NCs with an effective diameter of (a) $2.0 \mathrm{~nm}$, (b) $2.8 \mathrm{~nm}$, (c) $3.7 \mathrm{~nm}$, and (d) $6.2 \mathrm{~nm}$.

(110), and (112) reflections for all of these samples $\left(D=2.8^{-}\right.$ $6.2 \mathrm{~nm}$ ) confirmed the phase purity of the NCs to be ZB CdSe despite variation of the synthetic methods used to prepare these materials. Optical absorption and emission spectra in solution for each of the seed sizes synthesized (Fig. S3a $\uparrow$ ) exhibited a progressive red shift in the absorption and emission bands with increasing NC size. It should be noted that in addition to the expected stokes shifted emission for the $2.0 \mathrm{~nm}$ sample at $485 \mathrm{~nm}$, a significant broad low energy emission tail was also observed, which was attributed to surface-trap state emission. ${ }^{58}$ From these samples and optical measurements, a familiar exponential relationship of increasing optical band gap with decreasing NC particle size was observed over the particle size range from 2-8 nm (Fig. S3b $\dagger$ ). The ability to reproducibly and precisely control the size of ZB CdSe NCs was the critical first step in the synthesis of CdSe@CdS TP with controlled energetics.

\section{Preparation and characterization of uniform CdSe@CdS tetrapods}

A major challenge for the synthesis of monodisperse CdSe@CdS tetrapods was the strict need for ZB CdSe NCs to maintain their crystallographic phase purity both prior to, and during, W CdS arm growth, which becomes increasingly challenging with decreasing particle size. ${ }^{38,40,41,59}$ A major technical challenge that remained in the preparation of CdSe@CdS TPs was the ability to fully map the transition between type I $v s$. quasi-type II energetics by preparing uniform model tetrapods where only the size of the ZB CdSe was systematically varied from 2-6 $\mathrm{nm}$.

This synthetic target has been historically challenging since ZB CdSe has been shown to partially convert to the 
more stable $\mathrm{W}$ phase at high temperature over time, which raised concerns about the use of these crystals as precursors to tetrapod heterostructures, especially at ultrasmall sizes. In addition to maintaining high phase purity of ZB CdSe QDs across the size regime studied, it was vital for synthesized tetrapods to be of highly uniform dimensions and morphology. Feldmann et al. experimentally verified that tetrapods with longer arm lengths exhibited better spatial connectivity and improved charge transport. ${ }^{7}$ Additionally, we have previously demonstrated varying degrees of charge separation in CdSe@CdS nanorods of differing lengths prepared from monodisperse W CdSe seeds, ${ }^{48}$ and similar observations have been made for $4.0 \mathrm{~nm}$ seeded CdSe@CdS tetrapods by Feldmann. ${ }^{6,7}$ An attractive feature of the reported synthetic methods was the ability to prepare uniform CdSe@CdS TPs where the CdS arm lengths were precisely tuned over a range of 10-40 $\mathrm{nm}$ despite the variation of ZB CdSe seed sizes.

While the preparation of nanorod heterostructures from W CdSe NCs have been successful over a wide range of seed sizes and nanorod lengths using hot-injection methods, these approaches relied upon precise control over CdSe NC concentration. Unfortunately, when using ZB CdSe NC seeds to make CdSe@CdS TPs, the effective CdSe seed concentration was significantly lower (since $4 \mathrm{CdS}$ arms are grown in TPs, vs. 1 CdS "arm" in NRs), which was further exacerbated when using ultrasmall CdSe NC seeds. Hence, when attempting the synthesis of CdSe@CdS TPs using very small ZB CdSe NCs, competitive homonucleation of CdS NRs was also observed resulting in a mixture of CdS NRs and CdSe@CdS TPs (Fig. 2). In our previous work, we have demonstrated control of arm length for $6.0 \mathrm{~nm}$ seeded tetrapods synthesized from a one-pot hot-injection method, where CdS arm lengths were tuned between $20-120 \mathrm{~nm}$ for ZB CdSe seed concentrations ranging from 3.6-0.5 $\mu \mathrm{M}^{40,41}$ In the present study, we found that this worked for ZB CdSe NCs above $3.0 \mathrm{~nm}$ in size when the optical method of Hens was used to determine seed concentration and guide preparation of $1.7 \mu \mathrm{M}$ CdSe stock solu- tions in TOP. ${ }^{55}$ However, variations in arm length were observed between different seed sizes at identical calculated concentrations (up to $20 \%$ difference from $3.7 \mathrm{~nm}$ seeds $v s$. $6.2 \mathrm{~nm}$ seeds both at $1.7 \mu \mathrm{M}$ ), which we attributed to approximations in concentration determination and differing reactivities for different seed sizes. These approximations introduced significant error for seed sizes below $3.0 \mathrm{~nm}$, where seed reactivity was expected to be higher and thus slight errors in calculated concentration caused mixed product distributions. Thus, when this approach was applied to $2.0-2.8 \mathrm{~nm}$ ZB CdSe NCs at a calculated concentration of $1.7 \mu \mathrm{M}$, mixtures of tetrapods and tapered nanorods/nanorod fragments were observed, consistent with CdS homonucleation events (Fig. 2d). While one possible explanation for this would be impure ZB CdSe seeds (mixtures containing the W polytype), electron diffraction analysis of CdSe seeds in this size regime confirmed exclusively the formation of the ZB crystallographic phase (Fig. S8 $\dagger$ ). As a result, we posited that the observed product mixture seen in Fig. $2 \mathrm{~d}$ was attributed to global reduction of CdSe seed concentration due to the small seed sizes used in the TP reaction, which then allowed for kinetic competition of homonucleation of non-seeded CdS species and NR formation.

This issue was addressed by the development of an incremental injection approach for tetrapod synthesis from ultrasmall $2.0 \mathrm{~nm}$ ZB CdSe seeds (Fig. 2a), as opposed to the standard one-pot hot injection method (which can only be employed for larger ZB CdSe seed). In this approach, a 1.7 $\mu \mathrm{M}$ stock of $2.0 \mathrm{~nm} \mathrm{ZB}$ CdSe NCs (molecular weight determined by geometric approximations) was prepared in TOP, and upon incremental injection of sulfur-precursor $(0.55 \mathrm{M}$ $\mathrm{TOP}=\mathrm{S}$ ), overcoating of $\mathrm{ZB} \mathrm{CdSe}$ NCs with WZ CdS arms was progressively accomplished over the course of four injections, resulting in highly uniform CdSe@CdS tetrapods. By separating the $\mathrm{TOP}=\mathrm{S}$ injections into multiple sequential additions, the CdS precursor concentration was kept below the critical CdS homonucleation regime, and formation of CdSe@CdS

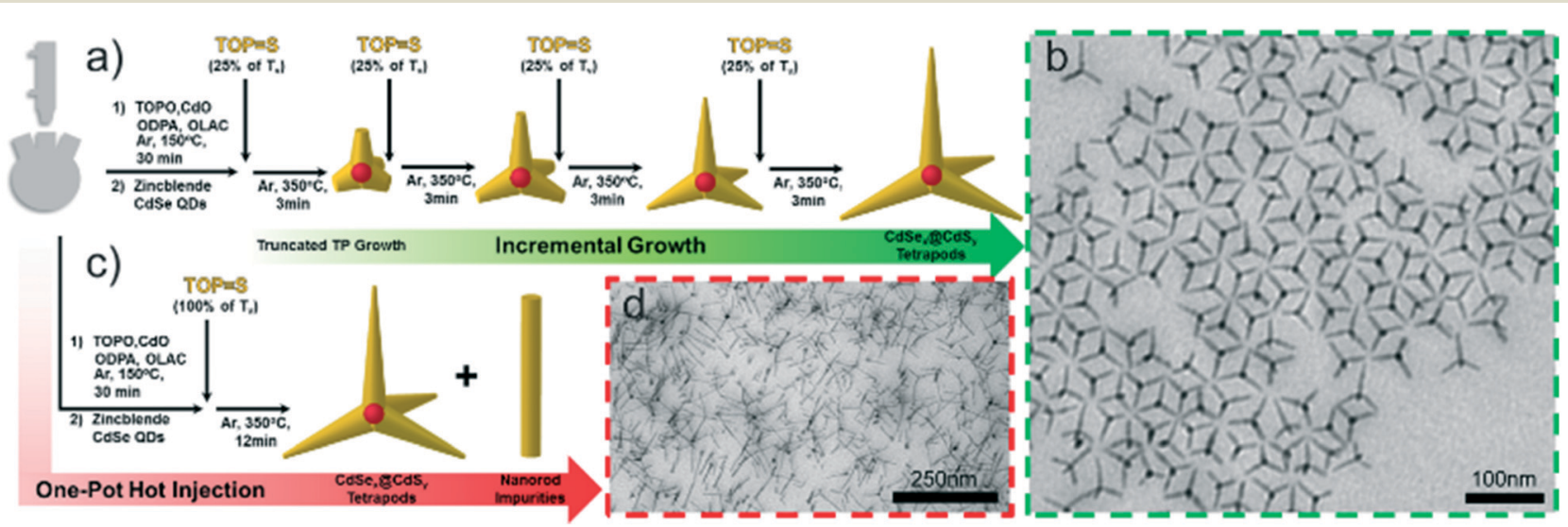

Fig. 2 (a) Schematic of the incremental injection approach utilized herein to prepare CdSe@CdS tetrapods from zincblende CdSe seeds of varying sizes, affording (b) uniform CdSeaCdS tetrapods that exhibit monolayer self-assembly as imaged by TEM (tetrapods shown are from $2.0 \mathrm{~nm}$ seeds). (c) Comparative schematic for the standard "hot injection" synthesis of CdSe@CdS tetrapod heterostructures, which yields (d) polydisperse tetrapods with significant nanorod impurities at small zincblende CdSe seed sizes as seen by TEM (products shown are from the same $2.0 \mathrm{~nm}$ seeds). 
heterostructures was observed as the exclusive product as determined by TEM imaging (Fig. 2b). Through kinetic studies, it was found that the ideal growth time between injections was 3 minutes, after which the next $\mathrm{TOP}=\mathrm{S}$ precursor injection would continue the growth of the tetrapods. We found that early time points were dominated by truncated tetrapod and cone-like species, which incrementally grew into tetrapods of $\sim 35 \mathrm{~nm}$ arm lengths by the end of the fourth injection as evidenced by over 95\% tetrapod heterostructures in the final product mixture before purification $(n=1225)$.

Furthermore, this method proved universal to all CdSe seed sizes utilized, providing access to uniform CdSe@CdS tetrapods of $\sim 35 \mathrm{~nm}$ arm lengths from $2.0 \mathrm{~nm}, 2.8 \mathrm{~nm}, 3.7$ $\mathrm{nm}$, and $6.2 \mathrm{~nm} \mathrm{ZB} \mathrm{CdSe} \mathrm{seeds} \mathrm{(Fig.} \mathrm{3).} \mathrm{Using} \mathrm{this} \mathrm{technique,}$ arm length was controlled through a binary weak/strong binding ligand system consisting of carboxylic/phosphonic

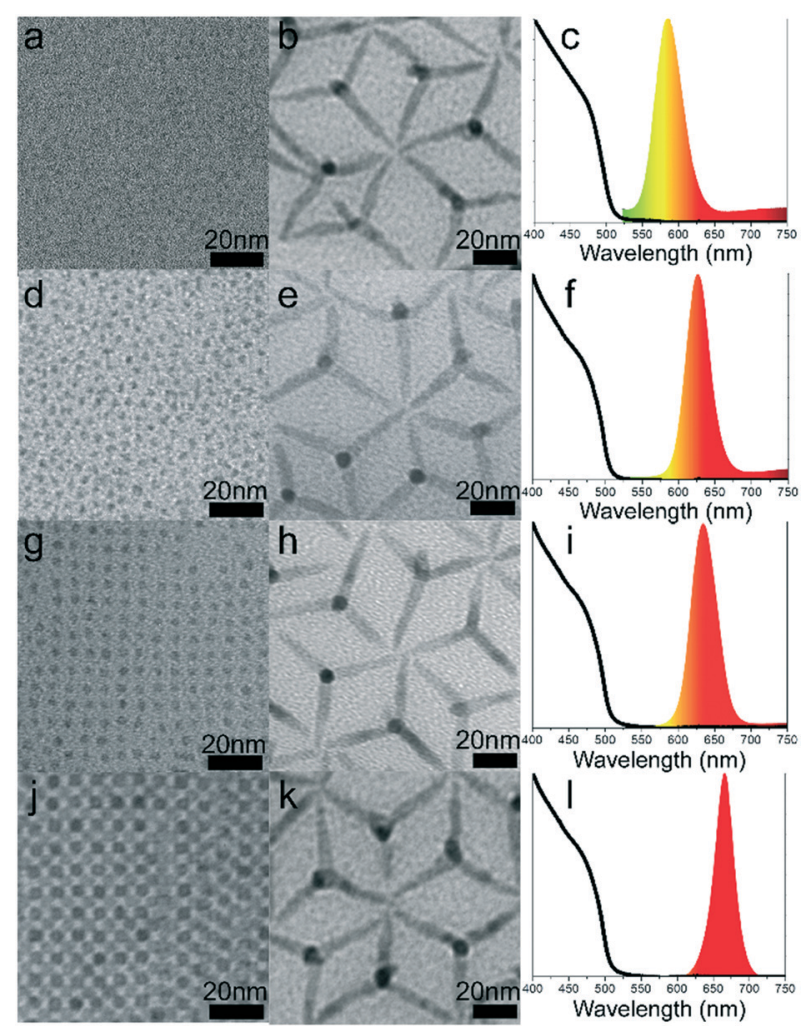

Fig. 3 Low magnification TEM images of zincblende CdSe NCs (column 1) used to seed the growth of CdSeaCdS tetrapods via the incremental injection approach (column 2), for which nearly identical absorption spectra are observed but progressive shifts in the emission maximum ( $\lambda_{\text {excitation }}=520 \mathrm{~nm}$ ) to lower energy with increasing seed size demonstrate the disparate CdSe core sizes in these otherwise identical NCs (column 3). Row $1(\mathrm{a}-\mathrm{c}$ ): The synthetic progression from (a) $2.0 \mathrm{~nm}$ zincblende CdSe seeds to (b) CdSeaCdS TPs with $32.0 \pm 5.5$ $\mathrm{nm}$ arms and (c) emission maximum at $587 \mathrm{~nm}$. Row 2 (d-f): The synthetic progression from (d) $2.8 \mathrm{~nm}$ zincblende CdSe seeds to (e) CdSe aCdS TPs with $35.0 \pm 4.2 \mathrm{~nm}$ arms and (f) emission maximum at $624 \mathrm{~nm}$. Row $3(\mathrm{~g}-\mathrm{i})$ : The synthetic progression from $(\mathrm{g}) 3.7 \mathrm{~nm}$ zincblende CdSe seeds to (h) CdSe@CdS TPs with $35.4 \pm 3.7 \mathrm{~nm}$ arms and (i) emission maximum at $635 \mathrm{~nm}$. Row 4 (j-l): The synthetic progression from (j) $6.2 \mathrm{~nm}$ zincblende CdSe seeds to (k) CdSe (CdS TPs with $36.2 \pm 3.9 \mathrm{~nm}$ arms and (l) emission maximum at $667 \mathrm{~nm}$. acids in $\sim 4: 1$ ratio, which previously has been demonstrated by Chan to affect final tetrapod dimensions. ${ }^{59}$ An attractive feature of the reported synthetic methods was the ability to prepare tetrapods of uniform length $(\sim 35 \mathrm{~nm})$ from a wide range of zincblende CdSe seeds $(2-6 \mathrm{~nm})$ at large scale (500$1000 \mathrm{mg})$. As confirmed through detailed manual sizings, each tetrapod sample exhibits a wide $(\sim 8-9 \mathrm{~nm})$ CdS base which tapers to a small diameter $(\sim 2-3 \mathrm{~nm})$ tip, regardless of CdSe seed size (Fig. S12 $\dagger$ ). To confirm the internal localization of the CdSe seed within the center of the tetrapod nanocrystals, spatially resolved energy dispersive X-ray analysis (EDX) studies were performed, for which characteristic Se-emission was only observed at the center of each of the tetrapod nanocrystals (Fig. S6 $\dagger$ ). In addition to structural uniformity, these tetrapods exhibited extensive long range monolayer ordering peculiar to such materials. Previously identified self-assembly mechanisms for tetrapods have highlighted the interdigitation of TP arms to form aggregated structures or "fishbone" type assemblies. ${ }^{59}$ For the materials reported herein, we observed two new types of assembly for TP nanocrystals, which can be classified as "tip-to-tip" (as seen in Fig. 2 and 3) or "tip-to-core" (as seen in minority in Fig. S4†). Preliminary experiments indicate a strong dependence of assembly on solvent polarity and processing conditions. In the work of Luitjen and coworkers, the effects of dipolar forces on tetrapod cluster assembly was studied in detail, wherein tip-to-core and tip-to-tip interactions were modeled to occur from favorable arm-to-arm interactions or charge stabilization by an external charged species, respectively. ${ }^{60}$ Presently, studies are underway in our lab to further elucidate and exploit the unique nature of these assemblies.

Despite comparable external dimensions, increasing CdSe seed size leads to a red shift in the seed emission observed for the tetrapods, indicative of the significant energetic differences that result from variation in CdSe core size (Fig. 3). Since CdS composes over 95\% of the volumetric fraction of all of the samples prepared, the absorption profiles of each sample appear nearly identical and characteristic of CdS as the primary light-absorbing component of the tetrapods. However, emission spectra of each of the tetrapods revealed primary emission from the respective $\mathrm{ZB}$ CdSe seed (Fig. 3c, f, i and l), confirming the strong electronic coupling between the light absorbing CdS arms and emitting CdSe core. Whereas slight shifts in emission are observed for the larger seed sizes $(3.7 \mathrm{~nm}$ and $6.2 \mathrm{~nm})$, dramatic red shifts were observed for samples below $3 \mathrm{~nm}$ relative to bare seeds ( $\sim 80 \mathrm{~nm}$ for $2.0 \mathrm{~nm}$ CdSe NCs). This was consistent with observations of quasi-type II behavior in CdSe@CdS nanorods, where increased electron delocalization into the CdS shell results in continuous red shifting and broadening of the emission signal with incremental shell growth. ${ }^{58,61-63}$

To further investigate the effect of seed size on CdSe@CdS tetrapod band alignment, transient absorption studies were carried out. In Fig. 4a, we compared the transient absorption spectra of CdSe@CdS tetrapods with seed sizes of $2.8 \mathrm{~nm}, 3.7$ $\mathrm{nm}$ and $6.2 \mathrm{~nm}$ (and arm lengths of $\sim 35 \mathrm{~nm}$ ) 1-2 ps after 


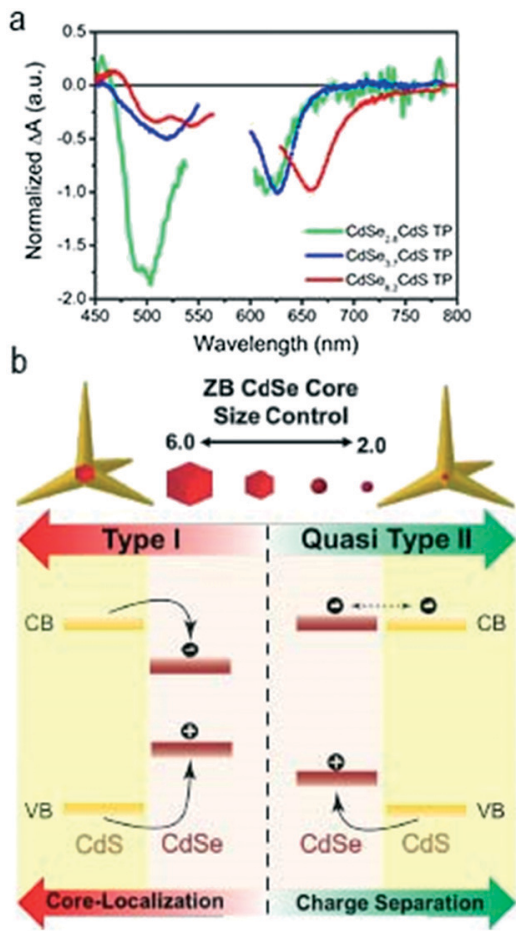

Fig. 4 (a) Transient absorption spectra of CdSeaCdS tetrapods, at 1-2 ps after selective excitation of the CdSe seed. Excitation wavelength: $580 \mathrm{~nm}$ for $2.8 \mathrm{~nm}$ and $4.0 \mathrm{~nm}$ seed, and at $590 \mathrm{~nm}$ for $6.2 \mathrm{~nm}$ seed. (b) Schematic representation of band edge alignment as a function of CdSe core size in tetrapods with identical CdS components. With decreasing size, the heterostructure band alignment shifts from type I to quasi-type II, resulting in enhanced charge separation across the heterostructure with smaller seed sizes.

selective excitation of the CdSe transition at 580,580 and 590 $\mathrm{nm}$, respectively. These excitations of the lowest energy exciton bands in these tetrapods produced an exciton at the band edge. For CdSe@CdS tetrapods with seed size of $2.8 \mathrm{~nm}$, the transient spectrum showed the bleach of the lowest energy exciton bands of both CdS arm (at $\sim 500 \mathrm{~nm}$ ) and CdSe seed (at $620 \mathrm{~nm}$ ). Furthermore, both bleach features form instantaneously and decay with the same kinetics (see Fig. S10†). Extensive previous transient absorption studies of CdSe, CdS and CdSe@CdS nanorods showed that these exciton bleach bands are caused by the state filling of the conduction band electron levels, with negligible contributions of valence band holes. $^{32,33,48,64}$ This result suggested that for CdSe@CdS tetrapods with a seed size of $2.8 \mathrm{~nm}$, the electron wavefunction in the lowest energy excitons extend from the CdSe seed into the CdS rod, confirming a quasi-type II band alignment (Fig. 4b). Interestingly, for CdSe@CdS tetrapods with larger seed sizes $(3.7 \mathrm{~nm}$ and $6.2 \mathrm{~nm})$, the transient absorption spectra in Fig. 4 showed only the bleach of the CdSe exciton band, without the pronounced CdS exciton bleach. A small exciton bleach at $\sim 520$ and $510-540 \mathrm{~nm}$ (for $3.7 \mathrm{~nm}$ and 6.2 $\mathrm{nm}$ seeds, respectively) can be attributed to higher energy transitions in the CdSe seed. This assignment was supported by comparison with transient spectra of CdSe quantum dots (seed only) shown in Fig. S13.† Thus for these tetrapods with larger seeds, the wavefunction of electrons in the lowest energy excitons is confined within the CdSe seed, with negligible extension into the CdS rod, which was consistent with the type I band alignment shown in Fig. 4.

\section{Seed size dependent Au-tipping kinetics}

Access to tetrapods with uniform dimensions that span quasi-type II/type I band alignments provided a unique opportunity to probe the effect of CdSe seed size on the chemical reactivity of the overall tetrapod heterostructures. While similar studies have been performed on CdSe@CdS nanorods, tetrapod systems have been demonstrated to have higher molar absorptivities than nanorod systems (owing to the four CdS light-absorbing arms), making them intriguing candidates for photocatalytic applications if charge separation can be tuned to the same level as has been reported for nanorod systems.

In our previous work, type I CdSe@CdS tetrapods were exposed to gold(III) chloride precursor in the presence of organic solvent, and over the course of 1 hour of continued irradiation at low temperature $\left(-4{ }^{\circ} \mathrm{C}\right)$, initially deposited small AuNPs were observed to ripen into a single, large $(D \sim 7 \mathrm{~nm})$ AuNP at the terminus of one of four CdS arms for each tetrapod. For this system, the initial AuNP deposition was perceived to proceed through mild sacrificial corrosion of CdS arm surfaces upon photoexcitation in the presence of $\mathrm{Au}^{3+}$ precursor, and the photoinduced electrochemical Ostwald ripening event proposed by Banin for nanorod systems provided rationale for the selectivity of the final $\mathrm{Au}-\mathrm{CdSe} @ \mathrm{CdS}$ TPs.

To investigate the effects of band-alignment on the chemical reactivities of tetrapod NCs prepared herein, a photoinduced Au-deposition reaction was performed. For both nanorod and more recently, tetrapod systems, irradiation in the presence of Au-precursor has been shown to result in deposition of AuNPs onto exposed CdS surfaces, for which selectivity can be tuned by reaction conditions and nanocrystal composition. ${ }^{36,40,41}$ With increasing ZB CdSe NC size, one might expect that Au-photodeposition processes would not proceed owing to recombination of photoexcited charge carriers in the seed region of the type I systems. However, we have previously demonstrated that CdSe@CdS tetrapods with $6 \mathrm{~nm} \mathrm{ZB}$ CdSe seeds (presumably possessing type I band alignment) were in fact able to facilitate reduction of small AuNPs $(D \sim$ $2 \mathrm{~nm}$ ) randomly on CdS arm surfaces upon $365 \mathrm{~nm}$ irradiation in the absence of reducing agent. ${ }^{40,41}$ We speculate that incomplete electron transfer from the CdS arms to the CdSe seeds upon excitation allows for slow Au-reduction at the surface of the tetrapod arms, despite the energetic driving force for charge transfer and recombination within the CdSe seed of these type I structures.

In the present study, under identical conditions, we investigated the effects of AuNP tipping on CdSe@CdS TPs with 2.0-6.2 nm ZB CdSe seeds. Remarkably, we observed a 20fold increase in rate of selective Au-deposition upon $365 \mathrm{~nm}$ 


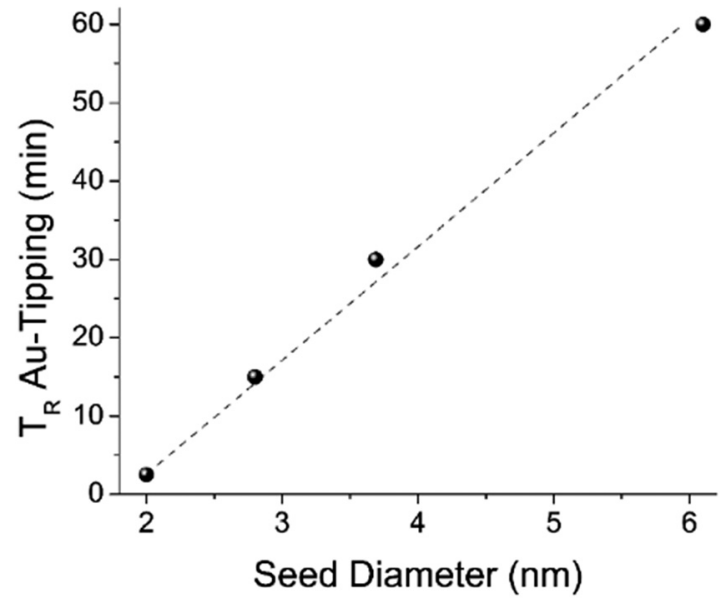

Fig. 5 Plot of time to achieve $7 \mathrm{~nm}$ diameter single AuNP tip as a function of CdSe seed diameter for small scale ( $8 \mathrm{mg}$ on Au-TP basis) $\mathrm{Au}-\mathrm{CdSe}$ CCdS TP synthesis under $365 \mathrm{~nm}$ irradiation, with smaller seed sizes resulting in rapid acceleration of reaction completion as monitored by TEM.

irradiation for quasi-type II CdSe@CdS TPs relative to type I TPs, where the synthesized TPs were identical with the exception of varying ZB CdSe seed sizes. Despite the energetic differences of the CdSe@CdS TPs, upon completion of the noble metal photodeposition, AuNP tips were converted to $7 \mathrm{~nm}$ in effective diameter, which allowed us to plot the effect of the $\mathrm{ZB}$ CdSe seed size $v s$. the final time required to grow a $7 \mathrm{~nm}$ AuNP tip. A linear trend was observed in final reaction time as a function of CdSe core size of the TP, as monitored by TEM, with reaction time decreasing proportionally with decreasing seed size (Fig. 5). In general, the rate acceleration observed was in agreement with increased charge separation between CdS/CdSe components with decreasing core size, which was expected to result in faster tipping kinetics for quasi-type II materials (Scheme 2). The linear nature of the observed trend was surprising and will be the subject of subsequent studies on the mechanism of AuNP deposition and ripening processes as they relate to specific valence band, conduction band, and trap state energy levels. To further investigate the origins of the observed rate enhancement for Au-photodeposition, TEM imaging of aliquots removed from the reaction mixture at varying times was conducted (Fig. 6). For $2.0 \mathrm{~nm}$ seeded tetrapods, early reaction times (under 30 seconds) revealed the formation of small AuNPs $(D=1.8 \pm$ $0.2 \mathrm{~nm}$ ) randomly decorating the CdS surfaces, which were observed to ripen into a single large $\operatorname{AuNP}(D=7.0 \pm 0.5 \mathrm{~nm})$ over the course of the subsequent 2 minutes (Fig. 6b-e). This trend was also supported by optical spectroscopy of the kinetic time points, wherein progressive AuNP growth was observed in the development of the size-dependent Au-SPR band between 550-600 $\mathrm{nm}$ (Fig. 6f). These observations are in strong agreement with the kinetic trends observed for photodeposited Au-CdSe@CdS NRs. ${ }^{34,39}$

This study revealed that the selective AuNP deposition onto CdSe@CdS TPs consisted of a two-step process: (i) initial deposition of small AuNPs on the CdS surface, which can only be initiated through reduction of $\mathrm{Au}^{3+}$ species by photoexcited electrons in the CdS arms, and (ii) the subsequent ripening of small AuNPs into a single, large AuNP through dissolution of less redox stable small AuNPs in favor of redeposition to form a more energetically stable, large AuNP (Fig. 6). ${ }^{39-41}$ Previously Banin had studied Auphotodeposition on nanorod systems, where the important role of amine capping ligands in the selective deposition of AuNPs onto nanorods of varying lengths was highlighted. ${ }^{34}$

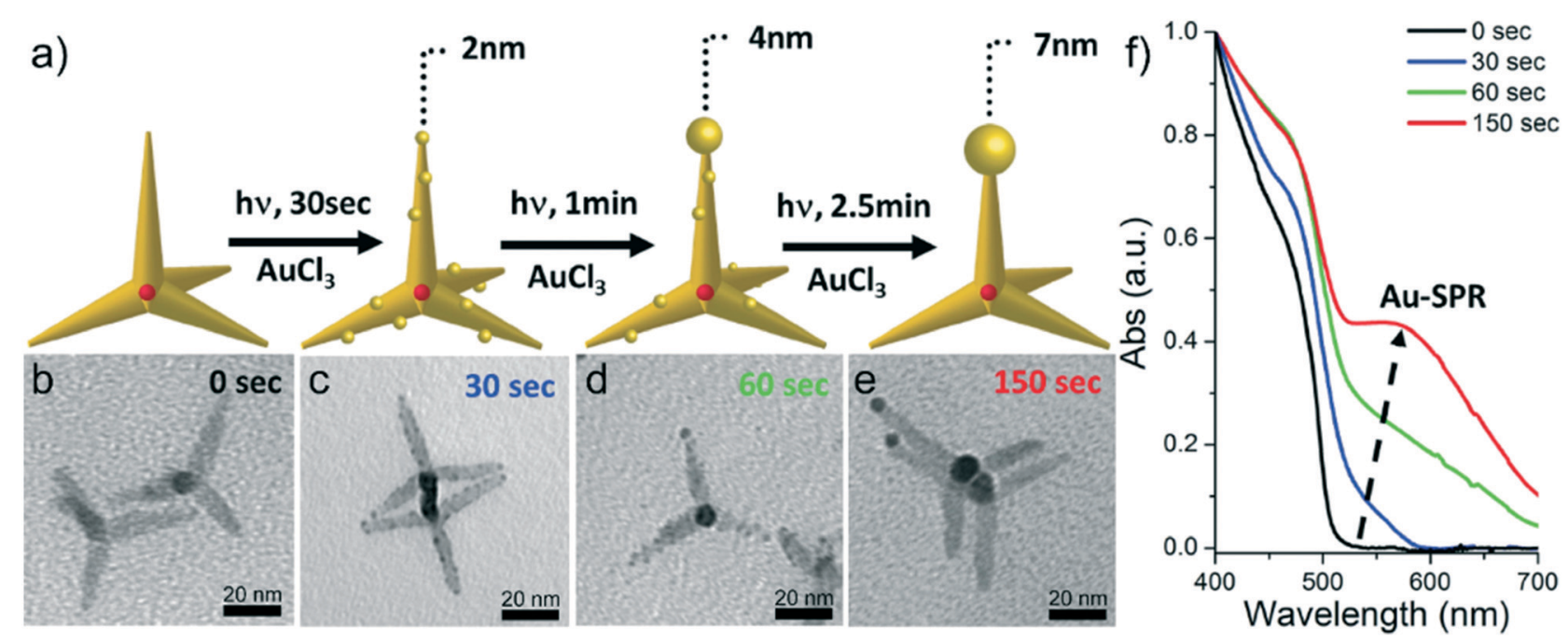

Fig. 6 Kinetic progression of small-scale Au-CdSeaCdS tetrapod synthesis from $2.0 \mathrm{~nm}$ seeded tetrapods under $365 \mathrm{~nm}$ UV-irradiation. (a) Schematic representation of kinetic product distributions with corresponding TEM images shown below at (b) 0 seconds, (c) 30 second, (d) 60 second, and (e) 150 seconds. (f) Absorption spectra of kinetic aliquots further supports the observed reaction kinetics, wherein the progression from TPs randomly decorated with small $(<2 \mathrm{~nm})$ AuNPs at short reaction times followed by rapid ripening to a single, large (7 nm) AuNP results in an AuSPR band of increasing magnitude and red-shifted character throughout the 2.5 minute reaction. 
However, in the present system, Au-deposition occurs onto CdSe@CdS TPs in the absence of reducing agents and was found to be solely dependent on irradiation. We therefore postulated that the initial AuNP deposition can proceed due to the ability of the CdSe@CdS tetrapods to undergo mild sacrificial photo-corrosion to facilitate $\mathrm{Au}^{3+}$ reduction and initial AuNP formation. Indeed, marginal corrosion of tetrapod surfaces was observed during the reaction $(\sim 5 \%$ of initial TP arm length), which was consistent with sacrificial oxidation of CdS species that would be expected for a reducing agent free synthesis due to uncompensated hole-buildup. In the presence of isopropanol, a known sacrificial donor for CdSe@CdS systems, identical reaction kinetics were observed as those described above, without the corrosion of tetrapod surfaces, further supporting this argument. Since this process relied on charge carrier generation and separation across the heterostructure upon irradiation, the rate of the Au-deposition processes in the smaller seeded tetrapods was enhanced owing to the quasi-type II band alignment of these samples.

Similar to initial AuNP deposition, the subsequent ripening process was expected to be highly dependent upon photoexcited charge carrier separation in the tetrapod heterostructure. Electrochemical Ostwald ripening processes have been shown to be driven by increasing redox stability of metallic nanoparticles with increasing size. ${ }^{65}$ In the work of Brus et al., when larger and smaller Ag-nanoparticles were shorted to each other through contact with a conductive substrate, they effectively functioned as cathode and anode in a galvanic cell, wherein sacrificial oxidation of smaller particles preceded reduction of the oxidized species onto larger nanoparticles. For hybrid metal-semiconductor NC systems, this was first observed by Banin et al., wherein four processes resulted in the ripening of randomly deposited AuNPs to a larger AuNP on the heterostructure surface: i) electrochemical reduction of Au-ions onto the NR surface, ii) oxidation of smaller, less redox stable AuNPs back into solution, iii) hopping of electrons left behind by Au-oxidation to a larger AuNP via surface states, and iv) Au-ion reduction onto the larger AuNP, resulting in a larger, more redox stable AuNP. ${ }^{34}$ In the present study, a similar effect was observed upon irradiation of tetrapods in the presence of gold precursor. Interestingly, complete AuNP ripening was only observed under irradiation, indicating the necessity of charge carrier generation for ripening processes to occur.

\section{Large scale AuNP photodeposition on quasi-type II tetrapods}

Synthetic accessibility to CdSe@CdS tetrapods with quasitype II band alignment from seed sizes below $3.0 \mathrm{~nm}$ enabled the rapid and scalable synthesis of highly asymmetric Janustype Au-CdSe@CdS tetrapods by direct utilization of photoexcited charge carriers for metal nanoparticle deposition and size-focusing. The ability to prepare multigram quantities of single AuNP tipped CdSe@CdS TPs was demonstrated using TPs containing $2.8 \mathrm{~nm} \mathrm{ZB}$ CdSe seeds, which was chosen for scale up due to both the synthetic accessibility of these colloi- dal precursors and the increased reactivity of these quasi-type II TPs toward photodeposition. While more reactive CdSe@CdS TPs with smaller $2.0 \mathrm{~nm}$ seeds were observed in the previous kinetic study, these smaller seeded TPs were more difficult to prepare and isolate on larger scale (i.e., greater than $1 \mathrm{~g}$ ). The mechanistic and structure-reactivity insights garnered from the earlier kinetic studies motivated the design of a scaled-up reaction of the photodeposition process reported above (300× larger than our earlier reports), where it was envisioned that more reactive CdSe@CdS TPs could be directly exposed to ambient sunlight (without any other photoirradiation) to provide the driving force to enable AuNP photodeposition. Upon exposure to sunlight at room temperature, rapid and selective tipping of $2.8 \mathrm{~nm}$ seeded tetrapods was observed (less than 90 seconds to reaction completion). Under these conditions, the sample was exposed to orders of magnitude greater photon flux across the visible spectrum, resulting in near-instantaneous gold deposition and ripening, as can be seen from the noticeably purple hue of the reaction mixture that develops in under two minutes of reaction time (Fig. 7). This particular demonstration to our knowledge represents the largest reaction scale for the preparation of any Janus-type metal tipped semiconductor nanocrystal (i.e., both metal tipped semiconductor nanorod or tetrapod). Recently, Amirav and coworkers have shown enhanced catalytic efficiency for metal and binary metal

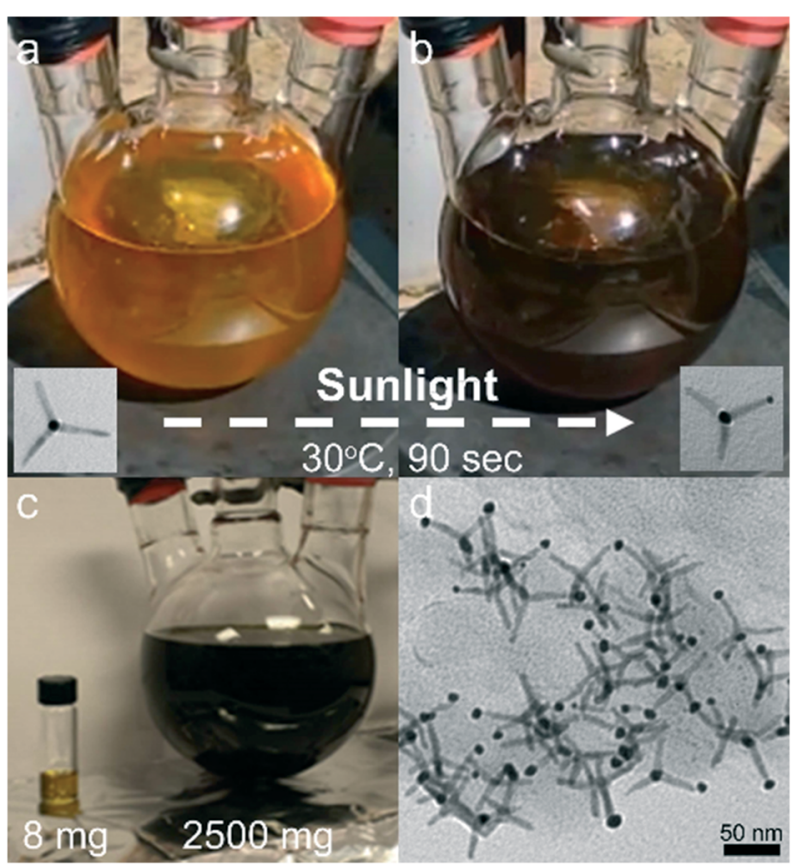

Fig. 7 The benefit of quasi-type II heterostructures: large scale synthesis of Janus Au-TP heterostructures from $2.8 \mathrm{~nm}$ seeded tetrapods. (a and b) $1.0 \mathrm{~g}$ of CdSeaCdS tetrapods synthesized from $2.8 \mathrm{~nm}$ zincblende CdSe seeds were observed to undergo selective AuNP deposition at scale at $30{ }^{\circ} \mathrm{C}$ in 90 seconds using only solar irradiation as the driving force, resulting in (c) a 300x scale-up of Janus Au-TP product nanoparticles $(2.5 \mathrm{~g})$ relative to standard scales for type I systems. (d) TEM image of Janus Au-CdSeaCdS TPs synthesized on 300× scale under solar irradiation. 
cocatalyst systems combined with CdSe@CdS heterostructured light absorbers. ${ }^{66-72}$ We envision the work presented herein to be a valuable contribution to such ongoing efforts towards practical, efficient, and high-yielding hydrogen evolution catalyst systems, having demonstrated the direct use of sunlight to prepare well defined metal-semiconductor nanocrystals at large scales.

\section{Conclusion}

In summary, we report on the use of a noble metal tipping reaction as a chemical probe to interrogate type I vs. quasi-type II energetics in CdSe@CdS TPs. Notably, synthetic methods to prepare highly stable ZB CdSe NCs ranging from $2.0-8.0 \mathrm{~nm}$ in size were developed to enable synthetic access to tetrapod nanocrystals for this study, which is the first example of fully mapping the effects of ZB CdSe seed sizes. These synthetic advances allowed the preparation of a series of well-defined, uniform CdSe@CdS TPs where the only variable in these materials was the sizes of the $\mathrm{ZB}$ CdSe seeds which allowed for further study of TP energetics and correlation with chemical reactivity toward AuNP photodeposition. To demonstrate the utility of such systems, a 300× scale up of Au-CdSe@CdS Janus nanoparticles was performed, allowing for selective Au-deposition in under 90 seconds using ambient Arizona sunlight as the sole driving force for metal deposition and ripening. Subsequent studies on these materials should focus on the detailed characterization of band-edge energetics, and utilization for visible light driven photocatalysis.

\section{Conflicts of interest}

There are no conflicts to declare.

\section{Acknowledgements}

This work was funded by the NSF (DMR-130792 for N. G. P., J. D., J. P.), the Division of Chemical Science, Geosciences and Biosciences, Office of Basic Energy Sciences of the U.S. Department of Energy (DE-FG03-02ER15753) (N. G. P., J. P.), U.S. Department of Energy (DE-SC0018285) (N. G. P., J. P.), the World Class University Program through the National Research Foundation of Korea funded by the Ministry of Education, Science and Technology (R31-10013) the NRF through the National Creative Research Initiative Center for Intelligent Hybrids (2010-0018290). T. L. is supported by the U.S. Department of Energy, Office of Science, Office of Basic Energy Sciences, Solar Photochemistry Program under Award Number (DE-FG02-12ER16347). The authors wish to thank Professor Neal Armstrong for helpful discussions throughout the course of the reported research.

\section{Notes and references}

1 J. I. Wong, N. Mishra, G. Xing, M. Li, S. Chakrabortty, T. C. Sum, Y. Shi, Y. Chan and H. Y. Yang, ACS Nano, 2014, 8, 2873-2879.
2 E. Shafran, N. J. Borys, J. Huang, D. V. Talapin and J. M. Lupton, J. Phys. Chem. Lett., 2013, 4, 691-697.

3 S. N. Raja, A. C. K. Olson, K. Thorkelsson, A. J. Luong, L. Hsueh, G. Chang, B. Gludovatz, L. Lin, T. Xu, R. O. Ritchie and A. P. Alivisatos, Nano Lett., 2013, 13, 3915-3922.

4 S. Liu, N. J. Borys, J. Huang, D. V. Talapin and J. M. Lupton, Phys. Rev. B: Condens. Matter Mater. Phys., 2012, 86, 045303.

5 C. L. Choi, H. Li, A. C. K. Olson, P. K. Jain, S. Sivasankar and A. P. Alivisatos, Nano Lett., 2011, 11, 2358-2362.

6 C. Mauser, E. Da Como, J. Baldauf, A. L. Rogach, J. Huang, D. V. Talapin and J. Feldmann, Phys. Rev. B: Condens. Matter Mater. Phys., 2010, 82, 081306.

7 A. A. Lutich, C. Mauser, E. Da Como, J. Huang, A. Vaneski, D. V. Talapin, A. L. Rogach and J. Feldmann, Nano Lett., 2010, 10, 4646-4650.

8 A. Fiore, R. Mastria, M. G. Lupo, G. Lanzani, C. Giannini, E. Carlino, G. Morello, M. De Giorgi, Y. Li, R. Cingolani and L. Manna, J. Am. Chem. Soc., 2009, 131, 2274-2282.

9 R. Costi, A. E. Saunders, E. Elmalem, A. Salant and U. Banin, Nano Lett., 2008, 8, 637-641.

10 R. Costi, G. Cohen, A. Salant, E. Rabani and U. Banin, Nano Lett., 2009, 9, 2031-2039.

11 A. Vaneski, J. Schneider, A. S. Susha and A. L. Rogach, APL Mater., 2014, 2, 012104.

12 C. L. Choi, K. J. Koski, S. Sivasankar and A. P. Alivisatos, Nano Lett., 2009, 9, 3544-3549.

13 W. Tie, Z. Jiagi, J. Lynch, C. Ou, W. Zhongliang, W. Xirui, D. LaMontagne, W. Huimeng, W. Zhongwu and Y. C. Cao, Science, 2012, 338, 358-363.

14 D. V. Talapin, J. H. Nelson, E. V. Shevchenko, S. Aloni, B. Sadtler and A. P. Alivisatos, Nano Lett., 2007, 7, 2951-2959.

15 L. Manna, D. J. Milliron, A. Meisel, E. C. Scher and A. P. Alivisatos, Nat. Mater., 2003, 2, 382-385.

16 L. Manna, E. C. Scher and A. P. Alivisatos, J. Am. Chem. Soc., 2000, 122, 12700-12706.

17 B. K. Hughes, J. M. Luther and M. C. Beard, ACS Nano, 2012, 6, 4573-4579.

18 Y. Xu, J. Lian, N. Mishra and Y. Chan, Small, 2013, 9, 1908-1915.

19 K. Manthiram, B. J. Beberwyck, D. V. Talapin and A. P. Alivisatos, J. Visualized Exp., 2013, e50731, DOI: 10.3791/ 50731, 50738 pp.

20 N. Mishra, J. Lian, S. Chakrabortty, M. Lin and Y. Chan, Chem. Mater., 2012, 24, 2040-2046.

21 J. Lian, Y. Xu, M. Lin and Y. Chan, J. Am. Chem. Soc., 2012, 134, 8754-8757.

22 J. Huang, M. V. Kovalenko and D. V. Talapin, J. Am. Chem. Soc., 2010, 132, 15866-15868.

23 L. Amirav and A. P. Alivisatos, J. Phys. Chem. Lett., 2010, 1, 1051-1054.

24 Y. Sung, J. Lim, J. H. Koh, L. J. Hill, B. K. Min, J. Pyun and K. Char, CrystEngComm, 2015, 17, 8423-8427.

25 E. Shaviv, O. Schubert, M. Alves-Santos, G. Goldoni, R. Di Felice, F. Vallee, N. Del Fatti, U. Banin and C. Soennichsen, ACS Nano, 2011, 5, 4712-4719. 
26 R. Costi, A. E. Saunders and U. Banin, Angew. Chem., Int. Ed., 2010, 49, 4878-4897.

27 A. E. Saunders, I. Popov and U. Banin, J. Phys. Chem. B, 2006, 110, 25421-25429.

28 L. Carbone, S. Kudera, C. Giannini, G. Ciccarella, R. Cingolani, P. D. Cozzoli and L. Manna, J. Mater. Chem., 2006, 16, 3952-3956.

29 A. Figuerola, I. R. Franchini, A. Fiore, R. Mastria, A. Falqui, G. Bertoni, S. Bals, G. Van Tendeloo, S. Kudera, R. Cingolani and L. Manna, Adv. Mater., 2009, 21, 550-554.

30 R. Lavieville, Y. Zhang, A. Casu, A. Genovese, L. Manna, E. Di Fabrizio and R. Krahne, ACS Nano, 2012, 6, 2940-2947.

31 R. Ehamparam, N. G. Pavlopoulos, M. W. Liao, L. J. Hill, N. R. Armstrong, J. Pyun and S. S. Saavedra, ACS Nano, 2015, 9, 8786-8800.

32 K. Wu, H. Zhu, Z. Liu, W. Rodriguez-Cordoba and T. Lian, J. Am. Chem. Soc., 2012, 134, 10337-10340.

33 K. Wu, Z. Chen, H. Lv, H. Zhu, C. L. Hill and T. Lian, J. Am. Chem. Soc., 2014, 136, 7708-7716.

34 G. Menagen, J. E. Macdonald, Y. Shemesh, I. Popov and U. Banin, J. Am. Chem. Soc., 2009, 131, 17406-17411.

35 A. Figuerola, M. van Huis, M. Zanella, A. Genovese, S. Marras, A. Falqui, H. W. Zandbergen, R. Cingolani and L. Manna, Nano Lett., 2010, 10, 3028-3036.

36 T. Mokari, E. Rothenberg, I. Popov, R. Costi and U. Banin, Science, 2004, 304, 1787-1790.

37 T. Mokari, C. G. Sztrum, A. Salant, E. Rabani and U. Banin, Nat. Mater., 2005, 4, 855-863.

38 E. Elmalem, A. E. Saunders, R. Costi, A. Salant and U. Banin, Adv. Mater., 2008, 20, 4312-4317.

39 G. Menagen, D. Mocatta, A. Salant, I. Popov, D. Dorfs and U. Banin, Chem. Mater., 2008, 20, 6900-6902.

40 N. G. Pavlopoulos, J. T. Dubose, N. Pinna, M.-G. Willinger, K. Char and J. Pyun, Angew. Chem., Int. Ed., 2016, 55, 1787-1791.

41 N. G. Pavlopoulos, J. T. Dubose, E. D. Hartnett, K. Char and J. Pyun, ACS Macro Lett., 2016, 950-954, DOI: 10.1021/ acsmacrolett.6b00511.

42 H. Eshet, M. Gruenwald and E. Rabani, Nano Lett., 2013, 13, 5880-5885.

43 L. T. Kunneman, M. Zanella, L. Manna, L. D. A. Siebbeles and J. M. Schins, J. Phys. Chem. C, 2013, 117, 3146-3151.

44 E. R. Smith, J. M. Luther and J. C. Johnson, Nano Lett., 2011, 11, 4923-4931.

45 N. J. Borys, M. J. Walter, J. Huang, D. V. Talapin and J. M. Lupton, Science, 2010, 330, 1371-1374.

46 D. Steiner, D. Dorfs, U. Banin, F. Della Sala, L. Manna and O. Millo, Nano Lett., 2008, 8, 2954-2958.

47 L. J. Hill, M. M. Bull, Y. Sung, A. G. Simmonds, P. T. Dirlam, N. E. Richey, S. E. DeRosa, I.-B. Shim, D. Guin, P. J. Costanzo, N. Pinna, M.-G. Willinger, W. Vogel, K. Char and J. Pyun, ACS Nano, 2012, 6, 8632-8645.

48 K. Wu, L. J. Hill, J. Chen, J. R. McBride, N. G. Pavlopolous, N. E. Richey, J. Pyun and T. Lian, ACS Nano, 2015, 9, 4591-4599.
49 M. G. Lupo, F. Della Sala, L. Carbone, M. Zavelani-Rossi, A. Fiore, L. Lueer, D. Polli, R. Cingolani, L. Manna and G. Lanzani, Nano Lett., 2008, 8, 4582-4587.

50 E. Yoskovitz, G. Menagen, A. Sitt, E. Lachman and U. Banin, Nano Lett., 2010, 10, 3068-3072.

51 A. Sitt, F. Della Sala, G. Menagen and U. Banin, Nano Lett., 2009, 9, 3470-3476.

52 S. N. Raja, D. Zherebetskyy, S. Wu, P. Ercius, A. Powers, A. C. K. Olson, D. X. Du, L. Lin, S. Govindjee, L.-W. Wang, T. $\mathrm{Xu}$, A. P. Alivisatos and R. O. Ritchie, Nano Lett., 2016, 16, 5060-5067.

53 M. R. Buck, J. F. Bondi and R. E. Schaak, Nat. Chem., 2012, 4, 37-44.

54 M. R. Buck and R. E. Schaak, Angew. Chem., Int. Ed., 2013, 52, 6154-6178.

55 R. Karel Čapek, I. Moreels, K. Lambert, D. De Muynck, Q. Zhao, A. Van Tomme, F. Vanhaecke and Z. Hens, J. Phys. Chem. C, 2010, 114, 6371-6376.

56 Y. A. Yang, H. Wu, K. R. Williams and Y. C. Cao, Angew. Chem., Int. Ed., 2005, 44, 6712-6715.

57 M. B. Mohamed, D. Tonti, A. Al-Salman, A. Chemseddine and M. Chergui, J. Phys. Chem. B, 2005, 109, 10533-10537.

58 B. O. Dabbousi, J. RodriguezViejo, F. V. Mikulec, J. R. Heine, H. Mattoussi, R. Ober, K. F. Jensen and M. G. Bawendi, J. Phys. Chem. B, 1997, 101, 9463-9475.

59 N. Mishra, W.-Y. Wu, B. M. Srinivasan, R. Hariharaputran, Y.-W. Zhang and Y. Chan, Chem. Mater., 2016, 28, 1187-1195.

60 D. W. Sinkovits and E. Luijten, Nano Lett., 2012, 12, 1743-1748.

61 S. Brovelli, R. D. Schaller, S. A. Crooker, F. GarcíaSantamaría, Y. Chen, R. Viswanatha, J. A. Hollingsworth, H. Htoon and V. I. Klimov, Nat. Commun., 2011, 2, 280-288.

62 I. Coropceanu, A. Rossinelli, J. R. Caram, F. S. Freyria and M. G. Bawendi, ACS Nano, 2016, 10, 3295-3301.

63 S. Christodoulou, G. Vaccaro, V. Pinchetti, F. De Donato, J. Q. Grim, A. Casu, A. Genovese, G. Vicidomini, A. Diaspro, S. Brovelli, L. Manna and I. Moreels, J. Mater. Chem. C, 2014, 2, 3439-3447.

64 H. Zhu and T. Lian, J. Am. Chem. Soc., 2012, 134, 11289-11297.

65 P. L. Redmond, A. J. Hallock and L. E. Brus, Nano Lett., 2005, 5, 131-135.

66 P. Kalisman, L. Houben, E. Aronovitch, Y. Kauffmann, M. Bar-Sadan and L. Amirav, J. Mater. Chem. A, 2015, 3, 19679-19682.

67 L. Amirav, F. Oba, S. Aloni and A. P. Alivisatos, Angew. Chem., Int. Ed., 2015, 54, 7007-7011.

68 E. Aronovitch, P. Kalisman, L. Houben, L. Amirav and M. Bar-Sadan, Chem. Mater., 2016, 28, 1546-1552.

69 E. Aronovitch, P. Kalisman, S. Mangel, L. Houben, L. Amirav and M. Bar-Sadan, J. Phys. Chem. Lett., 2015, 6, 3760-3764.

70 M. Wachtler, P. Kalisman and L. Amirav, J. Phys. Chem. C, 2016, 120, 24491-24497.

71 Y. Nakibli and L. Amirav, Chem. Mater., 2016, 28, 4524-4527.

72 P. Kalisman, Y. Kauffmann and L. Amirav, J. Mater. Chem. A, 2015, 3, 3261-3265. 